\title{
Apolipoprotein M: Research progress, regulation and metabolic functions (Review)
}

\author{
LI-ZHU HUANG ${ }^{1-3}$, JIA-LIN GAO ${ }^{3}$, CHUN PU $^{3}$, PU-HONG ZHANG ${ }^{3}$, \\ LI-ZHUO WANG ${ }^{1}$, GANG FENG ${ }^{3}$ and YAO ZHANG ${ }^{1,2}$ \\ ${ }^{1}$ Department of Biochemistry and Molecular Biology, Wannan Medical College, Wuhu, Anhui 241001; \\ ${ }^{2}$ Anhui Province Key Laboratory of Biological Macro-Molecules Research, Wannan Medical College, Wuhu, Anhui 242001; \\ ${ }^{3}$ Department of Endocrinology and Genetic Metabolism, Yijishan Hospital of Wannan Medical College, \\ Wuhu, Anhui 241002, P.R. China
}

Received June 23, 2014; Accepted March 16, 2015

DOI: $10.3892 / \mathrm{mmr} .2015 .3658$

\begin{abstract}
Apolipoprotein M (ApoM) is a novel lipoprotein-associated plasma protein of the apolipoprotein family. It is predominantly enriched in high-density lipoprotein (HDL), and is also present in small quantities in low-density lipoprotein (LDL) and in very low-density lipoprotein. Transgenic animal experiments have suggested that ApoM can be transformed into various lipoproteins and may be involved in lipoprotein metabolism. ApoM has five subtypes, however, their biological functions remain to be elucidated. The $\alpha$-helix, formed by ApoM through hydrophobic signal peptides, is anchored to the phospholipid monomolecular layers of HDL. Hydrophobic domains can associate with small lipophilic ligands and perform biological functions. ApoM may affect HDL metabolism and exhibit anti-atherosclerotic functions. Human HDL, containing ApoM subfractions, can protect LDL from oxidation and regulate cholesterol efflux more effectively than HDL without ApoM. Therefore, it is highly correlated with plasma cholesterol levels in the human body. Although previous studies have reported no difference in ApoM between groups of patients with coronary heart disease (CHD) and a normal control groups, the anti-atherosclerotic effect of ApoM is evident. ApoM is highly expressed in renal proximal tubule cells and is secreted into the urine in tubule cells. However, it is usually reabsorbed by giantin-associated proteins in a process, which is also affected in kidney disease. In addition to liver and kidney cells, low expression levels of ApoM occur in the intestinal tract and are associated with lymph node metastasis of colorectal cancer. ApoM gene polymorphism is associated with CHD, diabetes and other immune-associated diseases.
\end{abstract}

Correspondence to: Professor Yao Zhang, Department of Biochemistry and Molecular Biology, Wannan Medical College, 22 West Wenchang Road, Wuhu, Anhui 241001, P.R. China

E-mail: zhangyao@ahedu.gov.cn

Key words: apolipoprotein M, atherosclerosis, cholesterol efflux, high-density lipoprotein, cholesterol metabolism
Investigations into the gene regulation of ApoM may assist in further clarifying the role of ApoM in blood glucose and lipid metabolism. Genetic modification of the mouse ApoM gene is an essential technique to investigate the gene expression and regulation of ApoM, and to clarify the potential roles of ApoM in lipoprotein metabolism, atherosclerosis, diabetes and renal diseases.

\section{Contents}

1. Introduction

2. ApoM gene and protein

3. Regulation of ApoM

4. Association with diseases

5. Prospects

\section{Introduction}

Apolipoprotein M (ApoM), first identified by Xu Ning et al (1) in 1999, accounts for $~ 5 \%$ of high-density lipoprotein (HDL) and $<2 \%$ of low-density lipoprotein (LDL). It has a density similar to that of HDL2 (1.063-1.125 g/l) and HDL3 (1.125-1.210 g/l), and is one of the predominant components of HDL. The ApoM complex also contains HDL (apoA-I, apoA-II, apoC-I, apoC-II, apoC-III and apoJ) and LDL (apoB)-associated apolipoprotein (2). Plasma in adults has an ApoM content of 0.63-1.13 mmol/1 (3). ApoM is a newly identified protein with potential antioxidant activity and anti-atherosclerotic effects through cholesterol efflux (2). It has also been associated with diabetes, however, its specific biological functions and mechanisms of action remain to be elucidated. In the present review, the roles of ApoM gene regulation in lipid and HDL metabolism, and its association with the development of specific diseases are discussed, based on previous studies.

\section{ApoM gene and protein}

The ApoM gene is located on human chromosome 6p21.3, covers a span of $2.3 \mathrm{kbp}$ and is adjacent to the major 
histocompatibility complex (MHC) class III region. It is a single copy gene, containing six exons and five introns. The human ApoM protein has a molecular weight of $\sim 26 \mathrm{kD}$ and preferentially exists in HDL, followed by triglyceride-rich lipoprotein and LDL. Its structure is associated with the lipocalin family (1). Previous expression microarray analyses in multiple tissues (4) revealed that the expression of ApoM is highly tissue-specific and is specifically expressed in the liver and kidneys of humans. The mouse ApoM gene is located on chromosome 17 and covers a span of $2.8 \mathrm{kbp}$ (1). The sequence homology of the human and mouse ApoM genes is $\sim 79 \%$, and each contain six exons. ApoM is expressed in trace quantities in skeletal muscle and gastrointestinal tissues of human embryos at an early stage (3-5 months), and reaches a peak at 5-9 months (4). At the embryonic stage in mice, the expression of ApoM is detected at 7.5 days, peaks at 10.5 days and then begins to decline. Gradually, it becomes expressed, predominantly in the liver and renal tissues, and in trace quantities in heart, brain, spleen and testicular tissues of mouse embryos $(5,6)$. The biological function of ApoM during the embryonic period remains to be elucidated.

The first 20 amino acid residues of ApoM form a hydrophobic signal peptide $\alpha$-helix, which is retained in the mature protein, due to the lack of cleavage sites on the signal peptide. This feature is similar to two HDL apolipoproteins, paraoxonase (PON-1) and haptoglobin-related protein (7-9). The hydrophobic $\alpha$-helices of ApoM and PON-1 are anchored to HDL phospholipid monomolecular layers (9). Following mutation of Glu-22 of ApoM to Ala, a functional hydrolysis site is produced, leading to the hydrolysis of the signal peptide. In transgenic mice, mutant ApoM was no longer associated with lipoprotein and was rapidly filtered out through the kidney (9-11). In a previous study, in which HEK293 cells were stably transfected with the ApoM gene with a Q-22 mutation (10), culture of cells with wild-type ApoM in serum-free medium expressed ApoM in trace quantities compared with the cells with the ApoM mutation. The addition of serum or purified HDL stimulated the wild-type cell lines to secrete ApoM, which was further involved in the formation of HDL. However, in the HepG2 cell line, which synthesized HDL endogenously when cultured in serum-free medium, ApoM was only secreted in HDL-like particles, indicating that less ApoM was secreted without co-expression or exogenous addition of HDL to the medium. This may be due to the retention by the signal peptide. The results of this study confirmed that ApoM is an important component of HDL, and that HDL may promote the secretion of ApoM (10).

The apolipoprotein family, also termed the calycin-like protein family, has a common conservative structure consisting of a central core enclosed by an eight-stranded $\beta$-barrel structure, which is usually lined with hydrophobic or aromatic amino acid residues, to facilitate the association of hydrophobic molecules, including retinoic acid and 9-cis-retinoic acid $(6,12)$. Computer simulation imaging of the three-dimensional structure has indicated that ApoM has the same conserved structure as the apolipoprotein superfamily (13). The eight anti-parallel $\beta$-sheets of ApoM are connected by seven inserted loops $(13,14)$ and six cysteines form three disulfide bonds (13). There are two glycosylation sites at Asn-135 and Asn-148. Asn-135 is located in the periphery of the sheets and is a potential glycosylation site (13). In the three-dimensional model, characterized by an eight-stranded $\beta$-barrel structure, a fragment with Asn-135 may have an open or closed conformation (13).

Christoffersen et al (15) analyzed the crystal structure of amino acid residues 22-188 at the ApoM N-terminus, and demonstrated that 1-phospho-sphingosine (S1P) was bound to the 'calyx'-like ligand domain in the hydrophobic domain of the ApoM molecule. S1P hydrocarbon chains were observed to be close to the internal section of the calyx, indicating the interaction between S1P-ApoM and further involvement of the complex in HDL metabolism. Intrinsic tryptophan fluorescence analyses has indicated that, on the basis of structure and function, various subtypes of ApoM bind with vitamin A, all-trans retinoic acid and 9-cis-retinoic acid (15), supporting the concept of ApoM as an apolipoprotein. The above evidence suggests that S1P and retinoic acids are natural ligands of ApoM.

Plasma ApoM is primarily secreted from the liver, however, the ApoM expressed in the kidneys may only be metabolized in the kidneys (1). Studies have demonstrated that megalin mediates the intake of kidney-derived ApoM via the proximal tubules (16). Megalin is a membrane receptor, which is highly expressed in the renal proximal tubule and mediates the reabsorption of proteins, including lipid transfer proteins. Kidney-derived ApoM may be a ligand of megalin (16), suggesting that ApoM may be involved in the reabsorption of small molecular fat-soluble substances by renal tubules. LDL contains five subtypes of ApoM (17), including three $\mathrm{N}$-glycosylated and sialyated subtypes, one N-glycosylated, but not sialyated, subtype and one subtype without $\mathrm{N}$-glycosylation or sialyation. The former three subtypes account for $80-100 \%$ of the total ApoM in LDL. The latter subtype accounts for $20 \%$ of HDL and also contains five subtypes of ApoM, which can be separated by hydrophilic interaction chromatography (18). The significance of polymorphism of these ApoM subtypes in health and disease remains to be elucidated.

\section{Regulation of ApoM}

As the human ApoM gene is located at the MHC class III region (1), with multiple immune response-associated genes, it may be involved in the immune response. Xu et al (19) revealed that platelet-activating factor (PAF) significantly increased the mRNA expression levels and the secretion of ApoM in HepG2 cells. PAF or the PAF antagonist, lexipafant, selectively regulate the expression of ApoM. Subsequently, Xu et al (20) revealed that transforming growth factor (TGF) $\alpha$, epidermal growth factor and hepatocyte growth factor reduce the mRNA expression levels of ApoM and ApoB in HepG2 cells, however, to a lesser extent compared with TGF- $\beta$. These four cytokines had more significant effects on the mRNA expression levels of ApoM compared with the mRNA expression levels of ApoB. Hepatocyte nuclear factor (HNF)-1 $\alpha$ is an important transcription factor in the liver, kidney and pancreas. It is widely involved in cell growth, differentiation and metabolic processes (21). HNF-1 $\alpha$ binds to the promoter of ApoM and activates the transcriptional activity of the ApoM gene through conservative sites $(-103$ to -88$)$, thereby directly regulating the protein expression levels of ApoM (22). The 
expression of ApoM in the liver or renal tissues in the HNF-1 $\alpha$ gene knockout $\left(\mathrm{HNF}-1 \alpha^{-/}\right)$mice was minimal, whereas the levels of ApoM in the HNF-1 $\alpha$ transgenic $\left(\mathrm{HNF}-1 \alpha^{+/+}\right)$mice were 25 times higher. Disorders of bile acid transport in $\mathrm{HNF}-1 \alpha^{-/-}$mice may cause increased bile acid and cholesterol, therefore, affecting HDL metabolism (23). Mosialou et al (24) demonstrated that the pro-inflammatory substance, phorbol ester, can induce the AP-1 transcription factor at the ApoM promoter region (-53/-47), to recruit c-Jun and JunB protein kinase $\mathrm{C}$ associated proteins at $-214 /-14$, and competitively bind to the HNF-1 binding site of the ApoM promoter, thereby leading to the downregulation of ApoM.

Liver receptor homolog-1 (LRH-1) is important in the control of the early stage inflammatory reaction in hepatitis, reabsorption of intestinal crypt cells, bile acid biosynthesis, reverse cholesterol transport and other processes. Venteclef et al (25) demonstrated that ApoM was one of the target genes of LRH-1. LRH-1 binds to the LRH-1 response element in the proximal promoter region of ApoM (-83 to -67) and directly regulates the expression of ApoM in humans and mice. Liver $\mathrm{X}$ receptor (LXR) belongs to a liver receptor superfamily and is a ligand-activated transcription factor involved in regulation of inflammation and lipid metabolism (26). Zhang et al (27) revealed that LXR and its agonist, T0901317, can reduce the expression of ApoM in vivo and in vitro. Calayir et al (28) observed a decrease in the levels of ApoM in liver treated with T0901317, however, the expression levels of ApoM in the intestinal tissues and the adenosine triphosphate-binding cassette transporter (ABCA1) were increased. ABCA1 mediates cholesterol efflux to lipid-poor apoA-I, and ApoM can promote the formation of pre- $\beta$-HDL via ABCA1, which is a basic step for the formation of HDL (29). ABCA1 can regulate the expression of ApoM in HepG2 cells via the retinoid $\mathrm{X}$ receptor (RXR)/LXR pathway (30). Zhang et al (31) demonstrated that the expression of ApoM was reduced in HepG2 cells treated with 9-cis-retinoic acid and in combination with T0901317, can reduce the mRNA expression levels of ApoM in HepG2 cells by $65 \%$. However, several studies have suggested that LXR has multiple effects on ApoM. ApoM has high affinity for vitamin $\mathrm{A}$ and its derivatives, all-trans retinoic acid and 9-cis-retinoic acid (15). Mosialou et al (32) demonstrated that the levels of ApoM were significantly increased in HepG2 cells treated with 9-cis-retinoic acid. RXR $\alpha, \mathrm{HNF}-4$ and HNF-1, regulated by RXR, were all increased within 2-6 $\mathrm{h}$ following treatment, however, these returned to baseline levels within 8-24 h. HNF-4 $\alpha$ can bind to hormone response elements (HREs) at the proximal promoter (-33 to -21$)$ of ApoM. Following mutation in HRE, the primary activity of the ApoM promoter declines to $10 \%$ of the control group, and $\mathrm{HNF}-4 \alpha$-mediated transcription of the ApoM promoter is completely prevented. In addition to HNF- $4 \alpha$, a variety of receptor ligands of HREs can significantly induce the gene transcription of human ApoM and the activity of the ApoM promoter in HepG2 cells, and mutations in proximal HREs can lead to loss of function (32). Shih et al (23) demonstrated that HNF-1 regulates the expression of fragile $X$ mental retardation syndrome-related protein 1 (FXR-1) through nuclear receptor subfamily 1 , group $\mathrm{H}$, member 4 (Nr1h4) gene, encoding FXR-1, and thereby regulates the activities of Src homology region 2 domain-containing phosphatase-1

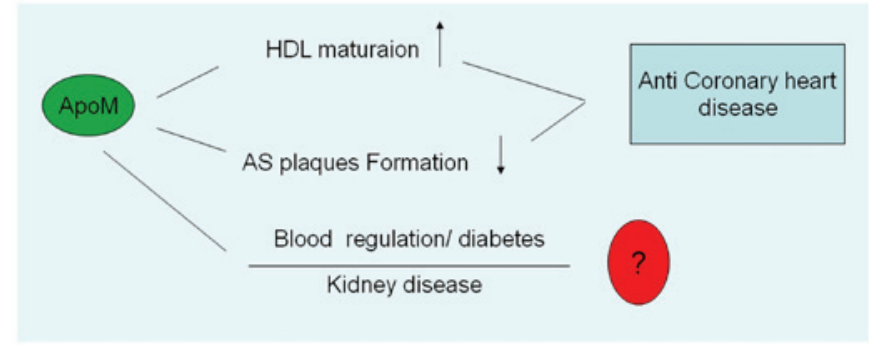

Figure 1. Involvement of ApoM in a several diseases. ApoM, apolopoprotein M; HDL, high-density lipoprotein; AS, atherosclerotic.

(SHP-1) and cholesterol $7 \alpha$-hydroxylase (CYP7A1). These results suggested that in bile acid and cholesterol metabolism, HNF-1 and FXR-1 may regulate ApoM through interactions with a number of factors. Venteclef et al (25) revealed that bile acids suppress the expression of ApoM in an SHP-dependent manner in vitro and in vivo, by inhibiting the transcriptional activity of LRH-1 on ApoM. It has also been confirmed (27) that, in HepG2 cells treated with HNF-1 $\alpha$ small interfering RNA, the levels of ApoM are significantly reduced, as are apoA-I, CYP7A1, FXR and SHP-1 (27). This suggests that, in addition to directly regulating ApoM, HNF-1 $\alpha$ may interact with other regulatory factors. Estrogen and androgen are also involved in the regulation of ApoM (33).

\section{Association with diseases}

Although the ApoM is predominant in HDL, the biological functions and mechanism of ApoM in HDL and disease remain to be elucidated. ApoM gene regulation has been reported in lipid and HDL metabolism. ApoM also participate in the formation of atherosclerotic plaques. ApoM is associated with diabetes and the development of kidney disease and other diseases (Fig 1).

Association with coronary heart disease (CHD). HDL particles have anti-atherosclerotic effects, predominantly by enabling the reversal of cholesterol transport to the liver, to be excreted along with bile (34-36). Mature HDL originates from nascent HDL, which is either produced by the liver or from the lipolysis of very low density lipoprotein (VLDL) and chylomicrons, along with fat-free ApoA-I or poor fat pre $\beta$-HDL (29). Intracellular cholesterol, transferred by pre $\beta$-HDL, is esterified by lecithin cholesterol acyltransferase (LCAT) from its mature form, which further promotes the maturation of pre $\beta$-HDL and increases the particle size $(37,38)$. Esterified cholesterol in mature HDL particles can be phagocytized by the liver cells via pinocytosis, and the triglycerides and cholesterol esters can be indirectly exchanged to LDL (39). The particle density of ApoM is close to that of HDL2 and HDL3. In vitro experiments have confirmed that HDL (ApoM+) is more effective as an antioxidant compared with HDL (ApoM-) (2), and promotes cholesterol efflux from THP-1 cells $(2,39,40)$. Furthermore, LDL (ApoM+) is more resistant to oxidation compared with LDL (ApoM-) (2). ApoM can affect HDL particle conversion through LCAT (41). It has been confirmed that ApoM enables pre $\beta$-HDL to be transformed into mature HDL 
particles, which are involved in cholesterol efflux and may have anti-atherosclerotic effects (42). In LDL receptor-deficient mice fed a high cholesterol diet, overexpression of the ApoM gene increases plasma concentrations of ApoM and delays the development of early atherosclerosis (42). In a previous study involving the treatment of C57BL/6 mice with ApoM siRNA, the expression of ApoM was reduced by $90 \%$ and HDL-c was reduced by $25 \%$. In addition, pre $\beta$-HDL was almost absent in $\mathrm{TCF}^{-/-}$mice and mice treated with TCF siRNA. In transgenic mice injected with HDL2 particles, large particle HDL1, rather than small particle HDL, was detected, suggesting that ApoM-HDL2 particles were transformed into pre $\beta$-HDL and promoted HDL maturation (37-39). In $\mathrm{LDLR}^{-/-}$mice injected with adenovirus-ApoM, the plasma levels of HDL were increased by $40 \%$ compared with control mice, the average incidence of atherosclerosis of the aortic root was reduced to $28 \%$ of the control group, and lesion areas, assessed using positive oil red $\mathrm{O}$ staining, of the large artery and aortic root, were reduced by $70 \%$. This suggested that ApoM protected the hypercholesterolemia $\mathrm{LDLR}^{-/-}$mice from atherosclerotic plaque formation (42). Although in vivo and in vitro investigations have indicated that ApoM exhibits anti-atherosclerotic effects, Ahnström suggested, through case control investigations, that ApoM levels in patients with CHD were unchanged compared with healthy control subjects (43). The effects of ApoM on human patients with CHD requires clarification in future experiments. Christoffersen reported that ApoM is not associated with CHD as it is, in part, associated with plasma LDL (40). In $\mathrm{LDLR}^{-/-}$mice, overexpression of ApoM led to increased levels of VLDL/LDL-c, and ApoM gene silencing led to decreased levels of VLDL/LDL-c. However, ApoM did not affect the levels of VLDL/LDL-c in mice with complete LDLR. In mice with an absence of functional LDLR, the ApoM-VLDL/LDL rich particles were removed and were at lower levels compared with the control VLDL/LDL particles, suggesting that ApoM decreased VLDL/LDL catabolism without depending on LDLR. ApoM reduced the atherosclerotic area in $\mathrm{ApoE}^{-/-}$mice and wild-type mice fed a high fat/high cholesterol diet. However, in $\mathrm{LDLR}^{-/-}$mice, the anti-atherosclerotic effect of ApoM was reduced, due to increased levels of VLDL/LDL. Loss of LDLR function led to increased levels of ApoM and VLDL/LDL, which reflected another aspect of the anti-atherosclerotic effects of ApoM. Christoffersen (40) also demonstrated a reciprocal interchange of ApoM between various lipoproteins in vivo. In wild-type mice, plasma ApoM was primarily associated with HDL particles, however, in transgenic mice, it was also observed in other abnormally elevated lipoproteins. In $\mathrm{LDLR}^{-/-}$mice, ApoM was enriched in LDL particles. In apoE ${ }^{-/-}$mice fed with normal diets, ApoM was predominantly present in HDL and was also detected in LDL and VLDL. In mice fed with a high fat/high cholesterol diet, ApoM was significantly increased in the VLDL of the intestine, but was only detected in trace quantities in HDL. The levels of HDL and ApoM levels in apoA- $\mathrm{I}^{-/}$mice were significantly lower compared with wild type mice. However, ApoM remained primarily in the HDL particles $(17,40,44,45)$. These findings suggest that additional investigations into the cross metabolic conversion of various lipoproteins are required to further improve understanding of the function of ApoM in lipid metabolism.
Elsøe et al $(46,47)$ observed cholesterol metabolism in peripheral cells in ApoM-knockout mice $\left(\mathrm{ApoM}^{-/}\right)$and ApoM-transgenic $\left(\right.$ ApoM $\left.^{-T g}\right)$ mice, and demonstrated that ApoM-HDL particles increased the efflux of ${ }^{3} \mathrm{H}$-labeled cholesterol from macrophages in the $\mathrm{ApoM}^{\mathrm{Tg}}$ mice by $>24 \pm 3 \%$ compared with the wild-type mice $(\mathrm{P}<0.05)$. Following intraperitoneal injection of foam cells with ${ }^{3} \mathrm{H}$-labeled cholesterols into $\mathrm{ApoM}^{-/-}$and $\mathrm{ApoM}^{-\mathrm{Tg}}$ mice, increased plasma ApoM did not significantly promote the transport of intracellular cholesterol to the plasma. However, following continuous intravenous infusion of ${ }^{13} \mathrm{C}$-2-labeled cholesterol/fat emulsion into $\mathrm{ApoM}^{-/}$, $\mathrm{ApoM}^{\mathrm{Tg}}$ and wild-type mice for $5 \mathrm{~h}$, the enrichment rate of free cholesterols and ${ }^{13} \mathrm{C}$-2-labeled cholesterol in the blood of the $\mathrm{ApoM}^{-\mathrm{Tg}}$ mice were lower compared with the $\mathrm{ApoM}^{-/-}$and wild-type mice. This suggested that levels of plasma ApoM promotes the mobilization of intracellular cholesterol transport in plasma. However, ApoM had no significant effect on the excretion of cholesterol into the feces.

The anti-inflammatory effects of ApoM may also be associated with its anti-atherosclerotic effects (48). In patients with sepsis with or without shock, and sepsis and infection with or without systemic inflammatory response syndrome, the average plasma levels of ApoM are all reduced, suggesting that ApoM can be used as a novel biomarker of sepsis $(49,50)$. The majority of S1P is bound to HDL in plasma, which increases the protective effects of HDL on endothelial cells (51). $\mathrm{S} 1 \mathrm{P}-\mathrm{ApoM}$ can activate the $\mathrm{S} 1 \mathrm{P}-1$ receptor and affect the functions of endothelial cells. S1P-ApoM is important in lipid metabolism, lipoprotein metabolism and the pathogenesis of atherosclerosis (52). These studies suggest novel directions for the investigation of ApoM. Through the overexpression and intraperitoneal injection of ${ }^{17} \mathrm{C}-\mathrm{S} 1 \mathrm{P}$, Makoto (53) demonstrated that ${ }^{17} \mathrm{C}$-S1P only existed in the liver cells and plasma, and was not present in the peripheral blood cells. Following the overexpression of ApoM in HepG2 cells and the mouse liver, the levels of S1P in the medium/plasma and peripheral blood cells/hepatocytes increased. Following adenoviral mediated overexpression of ApoM in hepatocytes, the levels of S1P in the plasma were increased, but no increase was observed in the peripheral blood cells. Further results indicated that S1P can associate with ApoM and affect the metabolism of S1P. Analysis of a single nucleotide of the proximal promoter region of the ApoM gene revealed that a single nucleotide $\mathrm{C}$ in ApoM T-778C, T-855C of the CHD group increased the risk of CHD, and the plasma total cholesterol levels in populations with CC and CT genotypes were significantly higher than a population with the TT genotype. These results suggested that polymorphisms of the ApoM gene promoter were risk factors for CHD (54-55). A previous meta-analysis (56) indicated that the ApoM T-778C polymorphism was associated with plasma LDL-c, and the levels of total cholesterol and LDL-c in $\mathrm{CT}+\mathrm{CC}$ groups were higher compared with those in the TT group. The $\mathrm{CT}+\mathrm{CC}$ genotypes exhibited a higher risk of CHD.

Association with diabetes. An analysis of 2,500 single nucleotide polymorphisms (SNPs), located in the MHC region, demonstrated that ApoM may be associated with the development of type 1 diabetes. ApoM was also revealed to interact with Kruppel-like factor 6, a tumor suppressor of prostate cancer, located on 10p15, and C8orf30A, a brain protein on 
$8 q 24$ of unknown function, which were not associated with type 1 diabetes. These results require further clarification in further investigations (57). Wu et al (58) revealed that an SNP of the T-778C polymorphic locus in the proximal promoter of ApoM was significantly associated with type 1 diabetes, and Zhou et al (59) demonstrated that T-778C was associated with cholesterol levels and susceptibility for type 2 diabetes, suggesting that T-778C of the ApoM gene promoter may be involved in the co-pathogenesis of type 1 and type 2 diabetes. The investigation of 17 SNPs of ApoM revealed that ApoM polymorphisms were associated with the susceptibility for type 2 diabetes in a population in Hong Kong, however, in patients who had suffered from the disease for $>10$ years, the rs805297C allele was significantly associated with type 2 diabetes and the rs707922 TT genotype significantly increased the levels of TC and LDL-C in patients with type 2 diabetes (59).

Leptin is a hormone-like protein, primarily secreted by fat cells, and ob is the protein product of the gene. Leptin is important in regulating food intake and energy metabolism in the body. It affects obesity, diabetes, cardiovascular disease, ischemia-reperfusion injury and other cellular metabolic pathways $(60)$. Xu et al $(61,62)$ performed a case control study on obese females and females with normal body weight, and demonstrated that ApoM was positively correlated with levels of leptin and negatively correlated with levels of cholesterol. Further results (63) have demonstrated that the in vivo expression levels of ApoM in ob/ob mice with leptin deficiency and $\mathrm{db} / \mathrm{db}$ mice with leptin receptor deficiency, were significantly lower compared with control groups. However, treatment with leptin significantly increased the expression levels of ApoM in ob/ob mice. Luo et al (64) demonstrated that in vitro treatment with recombinant leptin, at doses higher than the physiological dose (>100 ng/ml), inhibits the expression of ApoM in HepG2 cells in a dose-dependent manner, and leptin at physiological doses $(10 \mathrm{ng} / \mathrm{ml})$ can downregulate the expression of ApoM. ApoM may therefore be a significant independent predictor in the assessment of HDL apoA-I and apoA-II catabolism in overweight and obese male patients with insulin resistance (65). ApoM is positively correlated with PCSK9 of the LDLR pathway in populations with a lean body mass, however, it is not correlated in overweight or obese patients (66).

The pathogenesis of maturity-onset diabetes of the young (MODY) is primarily due to heterozygous mutations in the HNF-1 $\alpha$ gene, leading to a deficiency of insulin secretion in pancreatic $\beta$ cells of the islet. Richter (22) demonstrated that plasma concentrations of ApoM in patients with MODY3 were $36 \%$ lower than in the normal control group (0.65, vs. 1.0; $\mathrm{P}=0.02$ ), and that ApoM was deficient in $\mathrm{HNF}-1 \alpha^{-/-}$mice (22). In a case control study, Cervin (67) suggested that the levels of ApoM in females carrying the HNF-1 $\alpha$ P291fsinsC mutation were $10 \%$ lower than those in the control group, and that ApoM was not useful as a biomarker of the HNF-1 $\alpha$ mutation (67). However, Mughal (68) reported that the levels of ApoM in HNF1A-MODY patients were significantly lower compared with patients with type 1 diabetes. Detection of the levels of ApoM can differentiate HNF1 $\alpha$-MODY and type 1 diabetes, and thereby provide an experimental basis for further diagnosis and treatment. In a mouse model of alloxan-induced diabetes, the plasma levels of insulin and ApoM were significantly lower compared with mice injected with saline, and insulin treatment increased the expression of ApoM in the liver and kidney tissues (69). High blood sugar levels can significantly inhibit the in vivo and in vitro expression of ApoM (70). Plasma levels of ApoM in mice with alloxan-induced diabetes were lower compared with normal mice, however, insulin levels were elevated following short-term infusion of high glucose, and the levels of ApoM in mice with high blood sugar were significantly decreased $(69,70)$. In HepG2 cells, glucose and insulin reduced the levels of ApoM, suggesting that insulin had indirect regulatory effects on ApoM in vivo (70). In HepG2 cells, low doses of insulin and insulin-like growth factor 1 suppressed the levels of ApoM in the cells in a time- and dose-dependent manner. The insulin-induced downregulation of ApoM can be inhibited by the insulin-specific receptor inhibitor, AG1024, and the phosphatidylinositol 3-kinase (PI3-K) inhibitor, LY294002, and peroxisome proliferator-activated receptors $\beta / \delta$ agonists (GW501516) suppress the expression of ApoM in HepG2 cells. This suggests that ApoM can be regulated through the PI3-K pathway (71), and the above-mentioned findings suggest that insulin may regulate ApoM through the $\mathrm{PI} 3-\mathrm{K}$ pathway in vivo and in vitro. $\mathrm{PI} 3-\mathrm{K} / \mathrm{Akt}$ is an important signaling pathway for insulin metabolism. Wolfrum et al (42) demonstrated that insulin activates phosphorylated PI3-K/Akt and induces the phosphorylation of Ser-156 at conservative sites of hepatocyte nuclear factor Foxa2, which interacts with Akt in the PI3-K pathway and inhibits the Foxa2-dependent transcription pathway. A Foxa2 Thr-156 mutant can resist the Akt-mediated phosphorylation and Foxa2-dependent transcriptional inactivation. Foxa 2 is known to bind to the -474 to -462 region of the ApoM promoter to directly regulate ApoM. The functions of ApoM and its role in the development of diabetes require further investigation (72).

Association with other diseases. Human ApoM is specifically expressed in the liver and kidney (4). However, Luo et al (73) revealed that ApoM is expressed in human intestinal tissues in trace quantities and may be associated with lymph node metastasis of colorectal cancer. Gu et al (74) compared the serum levels of ApoM in 126 patients with hepatitis B virus (HBV) and 118 healthy controls, and demonstrated that the serum levels of ApoM in patients were 27\% higher compared with the control group. HBV increased the promoter activity of ApoM and increased the translation and expression of the gene. Plasma levels of ApoM in patients with hepatocellular carcinoma (HCC) are significantly higher compared with normal subjects, however, the mRNA expression levels of ApoM in cancer tissues are lower compared with adjacent tissues (75). In a mouse model of hepatic ischemia-reperfusion, a gradual increase in the mRNA expression levels of ApoM was observed within 0.5-3 h, and the levels of ApoM declined to baseline levels within 6-24 h, however, the function of ApoM in ischemia remains to be elucidated (76). The human ApoM gene is adjacent to the MHC class III region, which is closely associated with the pathogenesis of rheumatoid arthritis (RA). Single gene analysis of patients with RA and healthy controls revealed that allele A was associated with blood lipid disorders in patients with RA and ApoM C-1065A, and may increase the risk of atherosclerosis in patients with RA (77). The ApoM T-778C and C-1065A SNPs have been associated with high 
risk of stroke in the Chinese population (78), and the ApoM gene polymorphism has also been associated with emphysema and altered lung function (79).

Obstructive sleep apnea (OSA) may be a risk factor for CHD. Proteomic analysis has revealed that the protein expression levels of ApoM in patients with OSA are abnormal, and multivariate regression analysis indicated that ApoM was an independent risk factor for OSA (80). In addition, patients with metabolic syndrome exhibit lower plasma levels of ApoM, however, this cannot predict vascular media thickness (IMT). Plasma levels of ApoM in patients with MetS was reported to be $\sim 15 \%$ lower than in individuals without MetS $(\mathrm{P}=0.036)$, and multivariate regression analysis suggested that IMT was only associated with MetS ( $\mathrm{P}=0.05)(81)$. Ahnström et al (82) compared 343 patients with abdominal aortic aneurysm (AAA) with 214 healthy volunteers, and demonstrated that the levels of ApoA-I, apoB and ApoM in patients with AAA were significantly lower than the healthy controls. The levels of ApoA-I were 1.62 , vs. $2.08 \mathrm{~g} / 1$, levels of apoB were 0.91 , vs. $1.04 \mathrm{~g} / \mathrm{l}$ and the levels of $A$ poM were 0.72 , vs. $0.91 \mathrm{mmol} / \mathrm{l}$ (all $\mathrm{P}<0.0001$ ). However, multivariate analysis demonstrated that only apoA-I and apoB were associated with AAA. Ahnström et al (83) also demonstrated that the levels of Apo in patients with critical limb ischemia, a relatively severe type of atherosclerosis, were lower compared with those in the healthy control group. The levels of ApoA-I were 1.23, vs. $2.08 \mathrm{~g} / \mathrm{l}$, levels of apoB were 0.93 vs. $1.04 \mathrm{~g} / 1$ and levels of ApoM levels were 0.75 , vs. $0.91 \mathrm{mmol} / 1$ (all $\mathrm{P}<0.0001$ ). However, multivariate regression analysis revealed that only apoA-I was an independent risk factor for critical limb ischemia. In the kidneys of 11 Sprague Dawley rats, Xu et al (76) induced $45 \mathrm{~min}$ ischemia, with reperfusion $24 \mathrm{~h}$ after the ischemia, and demonstrated that plasma levels of urea nitrogen and creatine were significantly increased between 6 and $24 \mathrm{~h}$. The plasma levels of ApoM were increased significantly $45 \mathrm{~min}$ after ischemia and $2 \mathrm{~h}$ after reperfusion, and urine levels of ApoM were also significantly increased between 2 and $6 \mathrm{~h}$ after reperfusion. These results suggested that ApoM functions as an acute inflammatory protein and can serve as a marker of acute kidney injury.

\section{Prospects}

ApoM is important in the development of CHD. It can promote HDL maturation and reverse the formation of atheromatous plaques. Therefore, it can serve as a novel marker for the diagnosis and target for the treatment of CHD. In the pathogenesis of diabetes, the complex mechanisms of insulin and blood sugar regulation, and their association with the development of diabetes, remain to be elucidated. ApoM is highly expressed in kidney tissues, is a ligand for renal proteins and can prevent the loss of lipid molecules in kidneys. However, its role in kidney disease also remains to be elucidated and its physiological and pathological functions in renal tissues require further investigation.

\section{Acknowledgements}

The authors would like to thank the participants for their dedication of this study. This study was supported by grants from the Science and technology Project in Wuhu (no. 2013cxy04), the National Natural Science Foundation of China (no. 81200632) and Anhui Provincial Natural Science Foundation (to Yao Zhang, no. 1508085MH149).

\section{References}

1. Xu N and Dahlbäck B: A novel human apolipoprotein (apoM). J Biol Chem 274: 31286-31290, 1999.

2. Christoffersen C, Nielsen LB, Axler O, Andersson A, Johnsen AH and Dahlbäck B: Isolation and characterization of human apolipoprotein M-containing lipoproteins. J Lipid Res 47: 1833-1843, 2006

3. Axler O, Ahnström J and Dahlbäck B: An ELISA for apolipoprotein $\mathrm{M}$ reveals a strong correlation to total cholesterol in human plasma. J Lipid Res 48: 1772-1780, 2007.

4. Zhang XY, Dong X, Zheng L, et al: Specific tissue expression and cellular localization of human apolipoprotein $M$ as determined by in situ hybridization. Acta Histochem 105: 67-72, 2003

5. Zhang XY, Jiao GQ, Hurtig M, et al: Expression pattern of apolipoprotein $\mathrm{M}$ during mouse and human embryogenesis. Acta Histochem 106: 123-128, 2004.

6. Faber K, Axler O, Dahlbäck B and Nielsen LB: Characterization of apoM in normal and genetically modified mice. J Lipid Res 45: 1272-1278, 2004

7. Flower DR, North AC and Attwood TK: Structure and sequence relationships in the lipocalins and related proteins. Protein Sci 2: 753-761, 1993.

8. Sorenson RC, Bisgaier CL, Aviram M, Hsu C, Billecke S and La Du BN: Human serum Paraoxonase/Arylesterase's retained hydrophobic $\mathrm{N}$-terminal leader sequence associates with HDLs by binding phospholipids: apolipoprotein A-I stabilizes activity. Arterioscler Thromb Vasc Biol 19: 2214-2225, 1999.

9. Axler O, Ahnström J and Dahlbäck B: Apolipoprotein M associates to lipoproteins through its retained signal peptide. FEBS Lett 582: 826-828, 2008.

10. Duan J, Dahlbäck B and Villoutreix BO: Proposed lipocalin fold for apolipoprotein $\mathrm{M}$ based on bioinformatics and site-directed mutagenesis. FEBS Lett 499: 127-132, 2001.

11. Christoffersen C, Ahnström J, Axler O, Christensen EI, Dahlbäck B and Nielsen LB: The signal peptide anchors apolipoprotein $\mathrm{M}$ in plasma lipoproteins and prevents rapid clearance of apolipoprotein M from plasma. J Biol Chem 283: 18765-18772, 2008.

12. Flower DR, North AC and Sansom CE: The lipocalin protein family: structural and sequence overview. Biochim Biophys Acta 1482: 9-24, 2000.

13. Sevvana M, Ahnström J, Egerer-Sieber C, Lange HA, Dahlbäck B and Muller YA: Serendipitous fatty acid binding reveals the structural determinants for ligand recognition in apolipoprotein M. J Mol Biol 393: 920-936, 2009.

14. AhnströmJ,FaberK, AxlerO and Dahlbäck B:Hydrophobic ligand binding properties of the human lipocalin apolipoprotein M. J Lipid Res 48: 1754-1762, 2007.

15. Christoffersen $\mathrm{C}$, Obinata $\mathrm{H}$, Kumaraswamy SB, et al: Endothelium-protective sphingosine-1-phosphate provided by HDL-associated apolipoprotein M. Proc Natl Acad Sci USA 108: 9613-9618, 2011.

16. Faber K, Hvidberg V, Moestrup SK, Dahlbäck B and Nielsen LB: Megalin is a receptor for apolipoprotein $M$ and kidney-specific megalin-deficiency confers urinary excretion of apolipoprotein M. Mol Endocrinol 20: 212-218, 2006.

17. Karlsson $\mathrm{H}$, Lindqvist $\mathrm{H}$, Tagesson $\mathrm{C}$ and Lindahl $\mathrm{M}$ : Characterization of apolipoprotein $\mathrm{M}$ isoforms in low-density lipoprotein. J Proteome Res 5: 2685-2690, 2006.

18. Tetaz T, Detzner S, Friedlein A, Molitor B and Mary JL: Hydrophilic interaction chromatography of intact, soluble proteins. J Chromatogr A 1218: 5892-5896, 2011.

19. Xu N, Zhang XY, Dong X, Ekström U, Ye Q and Nilsson-Ehle P: Effects of platelet-activating factor, tumor necrosis factor and interleukin-1alpha on the expression of apolipoprotein $\mathrm{M}$ in HepG2 cells. Biochem Biophys Res Commun 292: 944-950, 2002.

20. XuN,HurtigM,Zhang XY,YeQ and Nilsson-EhleP:Transforming growth factor-beta down-regulates apolipoprotein M in HepG2 cells. Biochim Biophys Acta 1683: 33-37, 2004. 
21. Swenson ES, Guest I, Ilic Z, et al: Hepatocyte nuclear factor-1 as marker of epithelial phenotype reveals marrow-derived hepatocytes, but not duct cells, after liver injury in mice. Stem Cells 26: 1768-1777, 2008.

22. Richter S, Shih DQ, Pearson ER, et al: Regulation of apolipoprotein M gene expression by MODY3 gene hepatocyte nuclear factor-1alpha: haploinsufficiency is associated with reduced serum apolipoprotein M levels. Diabetes 52: 2989-2995, 2003

23. Shih DQ, Bussen M, Sehayek E, et al: Hepatocyte nuclear factor-1alpha is an essential regulator of bile acid and plasma cholesterol metabolism. Nat Genet 27: 375-382, 2001.

24. Mosialou I, Krasagakis K and Kardassis D: Opposite regulation of the human apolipoprotein M gene by hepatocyte nuclear factor 1 and Jun transcription factors. J Biol Chem 286: 17259-17269, 2011.

25. Venteclef N, Haroniti A, Tousaint JJ, Talianidis I and Delerive P: Regulation of anti-atherogenic apolipoprotein $\mathrm{M}$ gene expression by the orphan nuclear receptor LRH-1. J Biol Chem 283: 3694-3701, 2008.

26. Zhang Y and Mangelsdorf DJ: LuXuRies of lipid homeostasis: the unity of nuclear hormone receptors, transcription regulation, and cholesterol sensing. Mol Interv 2: 78-87, 2002.

27. Zhang Y, Chen CJ, Yang QL, Cheng LQ, Wang H and Huang LZ: Effect of interfering hepatocyte nuclear factor-1 alfa in HepG2 on the expressions of apoM, apoA-I and the correlative key enzyme of cholesterol metabolism. Zhonghua Gan Zang Bing Za Zhi 19: 121-126, 2011 (In Chinese).

28. Calayir E, Becker TM, Kratzer A, et al: LXR-agonists regulate ApoM expression differentially in liver and intestine. Curr Pharm Biotechnol 9: 516-521, 2008.

29. Fielding CJ and Fielding PE: Cellular cholesterol efflux. Biochim Biophys Acta 1533: 175-189, 2001

30. Di D, Wang Z, Liu Y, et al: ABCA1 upregulating apolipoproein M expression mediates via the RXR/lXR pathway in HepG2 cells. Biochem Biophys Res Commun 421: 152-156, 2012

31. Zhang X, Zhu Z, Luo G, Zheng L, Nilsson-Ehle P and Xu N: Liver $X$ receptor agonist downregulates hepatic apoM expression in vivo and in vitro. Biochem Biophys Res Commun 371: 114-117, 2008.

32. Mosialou I, Zannis VI and Kardassis D: Regulation of human apolipoprotein $\mathrm{m}$ gene expression by orphan and ligand-dependent nuclear receptors. J Biol Chem 285: 30719-30730, 2010.

33. Yi-Zhou Y, Guo Qing J, Jian Z, et al: Dihydrotestosterone regulating apolipoprotein $\mathrm{M}$ expression mediates via protein kinase $\mathrm{C}$ in HepG2 cells. Lipids Health Dis 11: 168, 2012.

34. Silver DL, Jiang XC, Arai T, Bruce C and Tall AR: Receptors and lipid transfer proteins in HDL metabolism. Ann NY Acad Sci 902: 103-111, 2000 .

35. Stein O and Stein Y: Atheroprotective mechanisms of HDL. Atherosclerosis 144: 285-301, 1999.

36. Assmann G and Nofer JR: Atheroprotective effects of high-density lipoproteins. Annu Rev Med 54: 321-341, 2003.

37. Phillips MC, Gillotte KL, Haynes MP, Johnson WJ, Lund-Katz S and Rothblat GH: Mechanisms of high density lipoprotein-mediated efflux of cholesterol from cell plasma membranes. Atherosclerosis 137 (Suppl): 13-17, 1998.

38. Santamarina-Fojo S, Lambert G, Hoeg JM and Brewer HB Jr: Lecithin-cholesterol acyltransferase: role in lipoprotein metabolism, reverse cholesterol transport and atherosclerosis Curr Opin Lipidol 11: 267-275, 2000.

39. Sparks DL and Pritchard PH: Transfer of cholesteryl ester into high density lipoprotein by cholesteryl ester transfer protein: effect of HDL lipid and apoprotein content. J Lipid Res 30 1491-1498, 1989

40. Christoffersen C, Pedersen TX, Gordts PL, Roebroek AJ, Dahlbäck B and Nielsen LB: Opposing effects of apolipoprotein $\mathrm{m}$ on catabolism of apolipoprotein B-containing lipoproteins and atherosclerosis. Circ Res 106: 1624-1634, 2010

41. Christoffersen C, Jauhiainen M, Moser M, et al: Effect of apolipoprotein $\mathrm{M}$ on high density lipoprotein metabolism and atherosclerosis in low density lipoprotein receptor knock-out mice. J Biol Chem 283: 1839-1847, 2008.

42. Wolfrum C, Poy MN and Stoffel M: Apolipoprotein M is required for prebeta-HDL formation and cholesterol efflux to HDL and protects against atherosclerosis. Nat Med 11: 418-422, 2005.

43. Ahnström J, Axler O, Jauhiainen M, et al: Levels of apolipoprotein $\mathrm{M}$ are not associated with the risk of coronary heart disease in two independent case-control studies. J Lipid Res 49: 1912-1917, 2008
44. Rye KA, Duong M, Psaltis MK, et al: Evidence that phospholipids play a key role in pre-beta apoA-I formation and high-density lipoprotein remodeling. Biochemistry 41: 12538-12545, 2002

45. Christoffersen C, Jauhiainen M, Moser M, et al: Effect of apolipoprotein $\mathrm{M}$ on high density lipoprotein metabolism and atherosclerosis in low density lipoprotein receptor knock-out mice. J Biol Chem 283: 1839-1847, 2008.

46. Elsøe S, Ahnström J, Christoffersen C, et al: Apolipoprotein M binds oxidized phospholipids and increases the antioxidant effect of HDL. Atherosclerosis 221: 91-97, 2012.

47. Elsøe S, Christoffersen C, Luchoomun J, Turner S and Nielsen LB: Apolipoprotein M promotes mobilization of cellular cholesterol in vivo. Biochim Biophys Acta 1831: 1287-1292, 2013.

48. Huang XS, Zhao SP, Hu M and Luo YP: Apolipoprotein M likely extends its anti-atherogenesis via anti-inflammation. Med Hypotheses 69: 136-140, 2007.

49. Christoffersen C and Nielsen LB: Apolipoprotein M-a new biomarker in sepsis. Crit Care 16: 126, 2012.

50. Kumaraswamy SB, Linder A, Akesson P and Dahlbäck B: Decreased plasma concentrations of apolipoprotein $M$ in sepsis and systemic inflammatory response syndromes. Crit Care 16: R60, 2012.

51. Sattler KJ, Elbasan S, Keul P, et al: Sphingosine 1-phosphate levels in plasma and HDL are altered in coronary artery disease. Basic Res Cardiol 105: 821-832, 2010

52. Arkensteijn BW, Berbée JF, Rensen PC, Nielsen LB and Christoffersen $C$ : The apolipoprotein m-sphingosine-1-phosphate axis: biological relevance in lipoprotein metabolism, lipid disorders and atherosclerosis. Int $\mathbf{J}$ Mol Sci 14: 4419-4431, 2013.

53. Kurano M, Tsukamoto K, Ohkawa R, et al: Liver involvement in sphingosine 1-phosphate dynamism revealed by adenoviral hepatic overexpression of apolipoprotein M. Atherosclerosis 229: 102-109, 2013

54. Xu WW, Zhang Y, Tang YB, et al: A genetic variant of apolipoprotein $\mathrm{M}$ increases susceptibility to coronary artery disease in a Chinese population. Clin Exp Pharmacol Physiol 35: 546-551, 2008.

55. Jiao GQ, Yuan ZX, Xue YS, et al: A prospective evaluation of apolipoprotein $M$ gene T-778C polymorphism in relation to coronary artery disease in Han Chinese. Clin Biochem 40: 1108-1112, 2007.

56. Zhang Z, Chu G and Yin RX: Apolipoprotein M T-778C polymorphism is associated with serum lipid levels and the risk of coronary artery disease in the Chinese population: a meta-analysis. Lipids Health Dis 12: 135, 2013.

57. Brorsson C, Hansen NT, Lage K, Bergholdt R, Brunak S, Pociot $\mathrm{F}$ and Diabetes Genetics Consortium: Identification of T1D susceptibility genes within the MHC region by combining protein interaction networks and SNP genotyping data. Diabetes Obes Metab 11 (Suppl 1): 60-66, 2009.

58. Wu X, Niu N, Brismar K, et al: Apolipoprotein M promoter polymorphisms alter promoter activity and confer the susceptibility to the development of type 1 diabetes. Clin Biochem 42: 17-21, 2009.

59. Zhou JW, Tsui SK, Ng MC, et al: Apolipoprotein M gene (APOM) polymorphism modifies metabolic and disease traits in type 2 diabetes. PLoS One 6: e17324, 2011.

60. Zhang YY, Proenca R, Margherita M, et al: Positional cloning of the mouse obese gene and its human homologue. Nature 372 425-432, 1994.

61. Ahima RS and Flier JS: Leptin. Annu Rev Physiol 62: 413-437, 2000.

62. Xu N, Nilsson-Ehle P and Ahrén B: Correlation of apolipoprotein $\mathrm{M}$ with leptin and cholesterol in normal and obese subjects. J Nutr Biochem 15: 579-582, 2004.

63. Xu N, Nilsson-Ehle P, Hurtig M and Ahrén B: Both leptin and leptin-receptor are essential for apolipoprotein $\mathrm{M}$ expression in vivo. Biochem Biophys Res Commun 321: 916-921, 2004.

64. Luo G, Hurtig M, Zhang X, Nilsson-Ehle P and Xu N: Leptin inhibits apolipoprotein $\mathrm{M}$ transcription and secretion in human hepatoma cell line, HepG2 cells. Biochim Biophys Acta 1734: 198-202, 2005

65. Ooi EM, Watts GF, Chan DC, et al: Association of apolipoprotein $\mathrm{M}$ with high-density lipoprotein kinetics in overweight-obese men. Atherosclerosis 210: 326-330, 2010.

66. Kappelle PJ,Lambert G, Dahlbäck B, Nielsen LB and Dullaart RP: Relationship of plasma apolipoprotein $\mathrm{M}$ with proprotein convertase subtilisin-kexin type 9 levels in non-diabetic subjects. Atherosclerosis 214: 492-494,2011. 
67. Cervin C, Axler O, Holmkvist J, et al: An investigation of serum concentration of apoM as a potential MODY3 marker using a novel ELISA. J Intern Med 267: 316-321, 2010.

68. Mughal SA, Park R, Nowak N, et al: Apolipoprotein M can discriminate HNF1A-MODY from Type 1 diabetes. Diabet Med 30: 246-250, 2013.

69. Xu N, Nilsson-Ehle P and Ahrén B: Suppression of apolipoprotein $\mathrm{M}$ expression and secretion in alloxan-diabetic mouse: Partial reversal by insulin. Biochem Biophys Res Commun 342: $1174-1177,2006$

70. Zhang $\mathrm{X}$, Jiang $\mathrm{B}$, Luo $\mathrm{G}$, Nilsson-Ehle $\mathrm{P}$ and $\mathrm{Xu} \mathrm{N}$ : Hyperglycemia down-regulates apolipoprotein $\mathrm{M}$ expression in vivo and in vitro. Biochim Biophys Acta 1771: 879-882, 2007.

71. Xu N, Ahrén B, Jiang J and Nilsson-Ehle P: Down-regulation of apolipoprotein $\mathrm{M}$ expression is mediated by phosphatidylinositol 3-kinase in HepG2 cells. Biochim Biophys Acta 1761: 256-260, 2006.

72. Wolfrum C, Howell JJ, Ndungo E and Stoffel M: Foxa2 activity increases plasma high density lipoprotein levels by regulating apolipoprotein M. J Biol Chem 283: 16940-16949, 2008.

73. Luo G, Zhang X, Mu Q, et al: Expression and localization of apolipoprotein $\mathrm{M}$ in human colorectal tissues. Lipids Health Dis 9: $102,2010$.

74. Gu JG, Zhu CL, Cheng DZ, Xie Y, Liu F and Zhou X: Enchanced levels of apolipoprotein M during HBV infection feedback suppresses HBV replication. Lipids Health Dis 10: 154, 2011.

75. Jiang J, Wu C, Luo G, et al: Expression of apolipoprotein M in human hepatocellular carcinoma tissues. Acta Histochem 113: 53-57, 2011.
76. Xu X, Ye Q, Xu N, et al: Effects of ischemia-reperfusion injury on apolipoprotein M expression in the liver. Transplant Proc 38: 2769-2773, 2006.

77. Park YJ, Yoo SA, Lee JH, Chung YJ, Cho CS and Kim W: The APOM polymorphism as a novel risk factor for dyslipidaemia in rheumatoid arthritis: a possible shared link between disease susceptibility and dyslipidaemia. Clin Exp Rheumatol 31: 180-188, 2013.

78. Zhao DX, He ZY, Qin X, et al: Association of apolipoprotein M gene polymorphisms with ischemic stroke in a Han Chinese population. J Mol Neurosci 43: 370-375, 2011.

79. Burkart KM, Manichaikul A, Wilk JB, et al: APOM and high-density lipoprotein cholesterol are associated with lung function and per cent emphysema. Eur Respir J 43: 1003-1017, 2014.

80. Kim J, Lee S, In K, et al: Increase in serum haptoglobin and apolipoprotein $\mathrm{M}$ in patients with obstructive sleep apnoea. J Sleep Res 18: 313-320, 2009.

81. Dullaart RP, Plomgaard P, de Vries R, Dahlbäck B and Nielsen LB: Plasma apolipoprotein $\mathrm{M}$ is reduced in metabolic syndrome but does not predict intima media thickness. Clin Chim Acta 406: 129-133, 2009.

82. Ahnström J, Gottsäter A, Lindblad B and Dahlbäck B: Plasma concentrations of apolipoproteins A-I, B and M in patients with abdominal aortic aneurysms. Clin Biochem 43: 407-410, 2010.

83. Ahnström J, Gottsater A, Lindblad B and Dahlbäck B: Plasma concentrations of apolipoproteins A-I, B and M in patients with critical limb ischemia. Clin Biochem 43: 599-603, 2010. 